\title{
Headache induced by isolated sphenoid fungal sinusitis: sinus headache?
}

\author{
Moon Oh Kwon · Kyung Soo Kim
}

Received: 14 July 2009/Accepted: 6 August 2009/Published online: 10 September 2009

(C) Springer-Verlag 2009

\begin{abstract}
In this article, we report a case of isolated sphenoid fungal sinusitis which only presented as headache. We review the literature of this rare but interesting lesion according to the revised 2004 IHS diagnostic criteria for "Headache attributed to Rhinosinusitis".
\end{abstract}

\section{Introduction}

The incidence and prevalence of noninvasive fungal sinusitis (fungus ball), broadly defined as any kind of sinonasal pathology related to the presence of fungi, are increasing [1]. Noninvasive fungal sinusitis is usually found in one sinus and the most frequently is caused by Aspergillus. The maxillary sinus is the most commonly involved site in the paranasal sinuses [2]. However, isolated sphenoid fungal sinusitis is a rare disease [3]. According to sphenoid sinus anatomy and depending on its contiguous structures, it is frequently difficult to diagnose, as patients present with various nonspecific symptoms and complications such as headache, visual disturbance and cranial nerve palsies [4]. Thus, diagnosis of the disease is typically not made until advanced imaging has been performed. Of these symptoms and complications, headaches are the most commonly present [5]. In this article, we report a case of isolated sphenoid fungal sinusitis which only presented as headache. We review the literature of this rare but interesting lesion according to the revised 2004

M. O. Kwon · K. S. Kim ( $\square)$

Department of Otorhinolaryngology-Head and Neck Surgery,

College of Medicine, Chung-Ang University Hospital, 224-1,

Heukseok-dong, Dongjak-gu, Seoul 156-755, Korea

e-mail: 99-21045@hanmail.net
IHS diagnostic criteria for "Headache attributed to Rhinosinusitis".

\section{Case report}

A 67-year-old female was referred to our department by a neurologist. She had a 2-month history of headache, which was intermittent, throbbing and of deep-seated character. The headache was located in retro-orbital area and extended to occipital area. It remained unchanged on standing, walking, bending, or coughing. There was no phono/photophobia or autonomic symptoms. She had no history of sinusitis or other sinus problems and also denied sinonasal symptoms such as nasal discharge, postnasal drip and nasal obstruction. Conventional analgesics and various drugs were prescribed by the neurologist in the past 2 weeks but had no effect. She did not have any medical disease. Physical examination revealed no abnormalities and no significant evidences of sinonasal tract infection. Upon neurological examination, no pathological localizing signs were observed. All the routine hematological and serological tests were within normal limits.

On examination of the nasal cavity, there were no intranasal abnormalities. A CT scan revealed a heterogeneous opacity with microcalcification involving the right sphenoid sinus (Fig. 1). On MRI, low signal intensity on T1 and a signal void on T2-weighted images were found (Fig. 2). A diagnosis of isolated sphenoid fungal sinusitis was made and the patient underwent an endoscopic sinus surgery. A wide right-sided sphenoidotomy was done and a freely movable brownish mass was found in the sinus with hypertrophied mucosa of the sinus. Fungal smear was positive and the histopathological examination was suggestive of aspergillosis. The sinus mucosa did not show any 

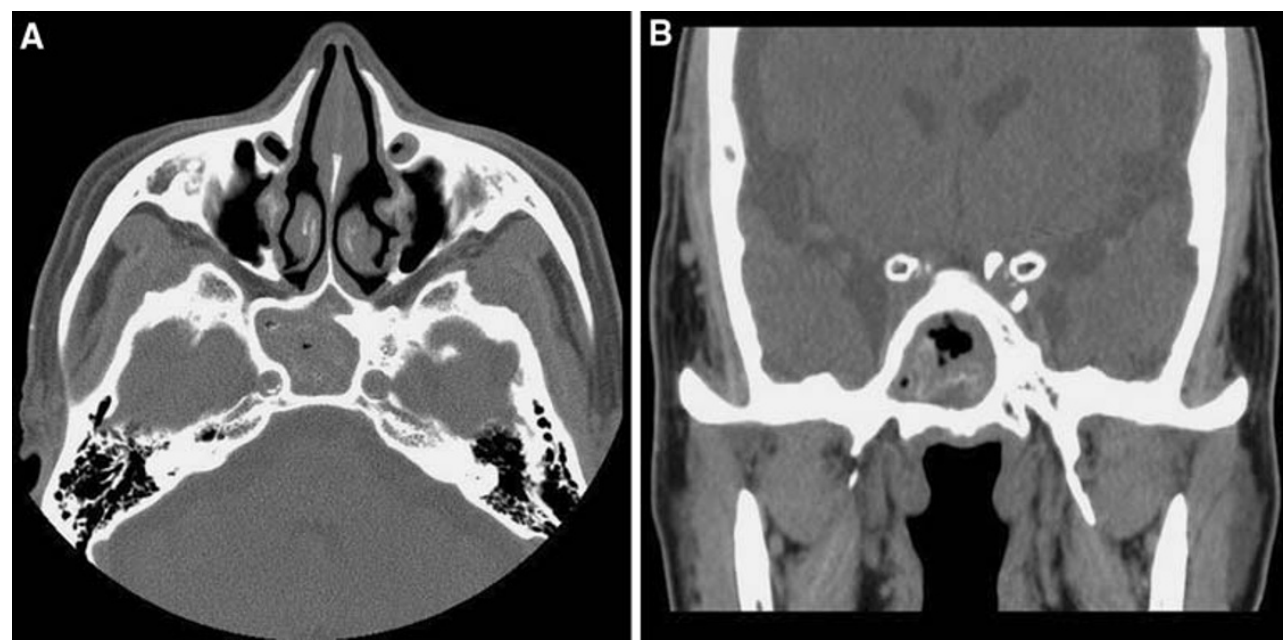

Fig. 1 Computed tomographic scans of the paranasal sinuses (a axial, b coronal) show a heterogeneous opacity with microcalcification involving the right sphenoid sinus

invasion by the fungus. Patient has been completely relieved of her symptoms for the 12 months and is on regular follow-up.

\section{Discussion}

The exact mechanism of spread of the fungal infection only to sphenoid sinus is not known [6]. Though not yet an established mechanism, fungal sinusitis will cause headache in a few patients, especially when the sphenoid sinus is involved [7]. Perhaps, because all of the paranasal sinuses are innervated by branches of the trigeminal sensory nerves, numerous noxious stimuli involving the sphenoid sinus and adjacent structures will trigger afferent sensory volleys into the trigeminal ganglion, resulting in the referred headache [8]. The headache location may be quite variable: retro-orbital, vertex occipital and diffuse headaches are the common presenting location [5, 6]. However, recent clinical studies have revealed that it is futile to attribute too much significance to headache localization resulting from sinus disease [8]. Thus, the 2004 IHS criteria removed this part in the diagnosis of sinus headache [7,9]. The typical headache caused by isolated sphenoid sinus lesion is usually described as transient, intermittent, deep seated, localized or throbbing character, and aggravated by standing, walking, bending, or coughing and often interferes with sleep because it tends to be worse in the evening $[6,10]$.

The issue of whether headaches are caused by sinonasal pathologies has caused much debate in the literature. The International Headache Society (IHS) has established criteria for "Sinusitis-related headache", and the revised International Headache Society classification (2004) which reiterates the requirement of clinical findings of acute sinusitis, along with a reversible "sinus headache", represents a very good reference also for ear, nose and throat (ENT) specialists and can be largely shared [9].

Although the headache character in our case is similar to sinus headache, we think that our patient does not fit the revised 2004 IHS diagnostic criteria of "Headache attributed to Rhinosinusitis" because of two important issues.

First, according to these criteria, it should have clinical evidences including purulence in the nasal cavity, nasal obstruction, hyposmia/anosmia and/or fever, developing simultaneously with onset or acute exacerbation of acute or acute-on-chronic rhinosinusitis in supporting the diagnosis of "sinus headache", However, this criterion may not be valid for isolated sphenoid sinus diseases, as nasal symptoms are lacking and the only major presenting symptom is headache. We could not find any clinical hints suggestive of sinus pathologies only presenting as headache without any rhinological symptoms in our case. Because the headache may precede sinus drainage in case of isolated sphenoid fungal sinusitis, clinical evidences as above stated, including purulent discharge are often lacking. Thus, isolated sphenoid sinus lesion, in the absence of sinonasal symptoms, usually delays its diagnosis.

Second, according to the revised 2004 IHS diagnostic criteria, chronic sinusitis is not validated as a cause of headache or facial pain unless it relapses into an acute stage. In our case, a fungal ball is a chronic and noninvasive form of fungal infection. Also, as above stated, we could not find any clinical symptoms suggestive of acute rhinosinusitis. Therefore, isolated sphenoid fungal sinusitis is not validated as a cause of headache if we strictly apply the revised 2004 IHS diagnostic criteria. However, our patient suffered from the headache induced 

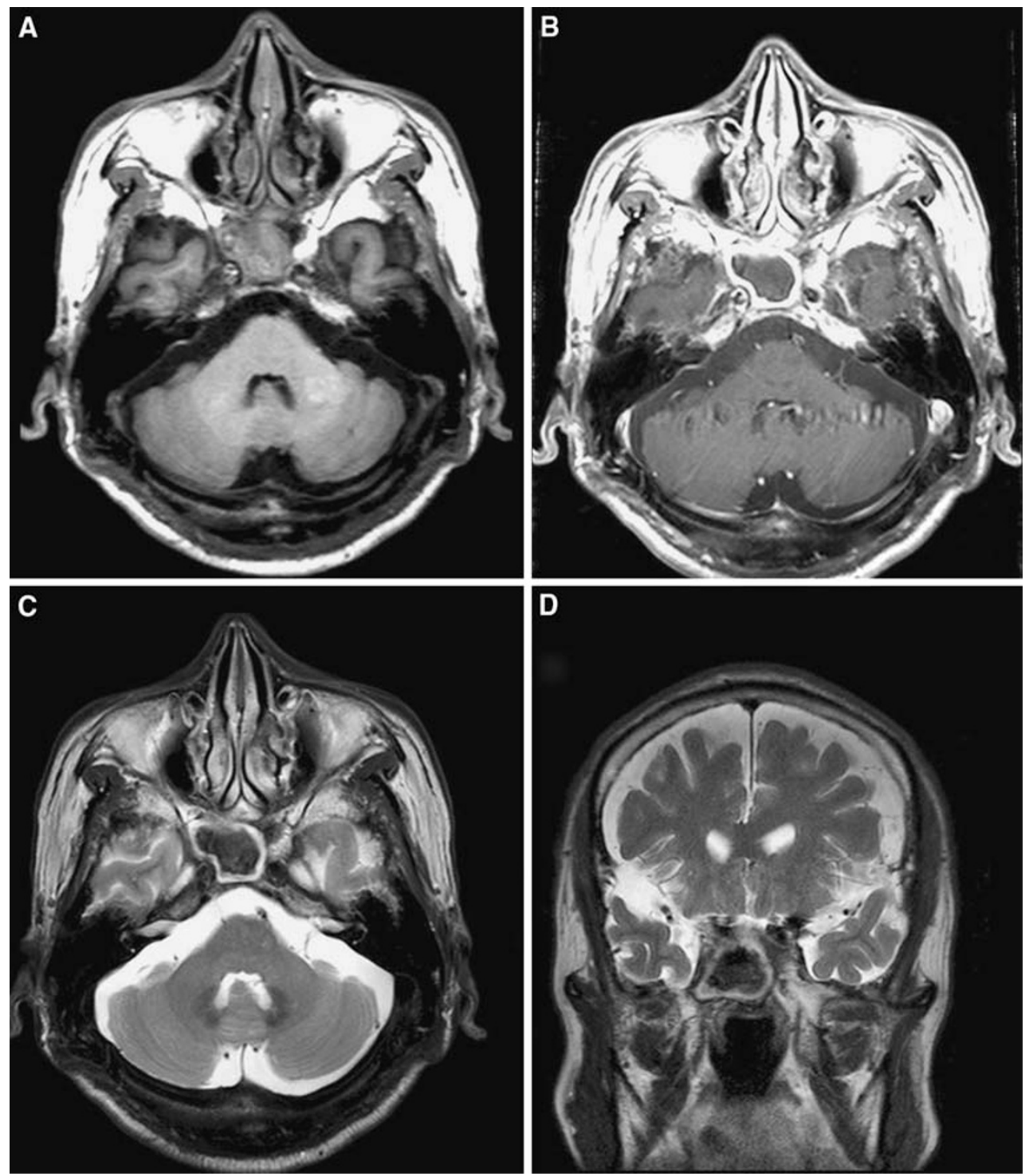

Fig. 2 The magnetic resonance imaging shows low signal intensity on T1 and a signal void on T2-weighted images in the right sphenoid sinus (a axial T1-weighted image, b axial gadolinium enhanced T1-weighted image, $\mathbf{c}$ axial T2-weighted image, $\mathbf{d}$ coronal T2-weighted image)

by isolated sphenoid fungal sinusitis for quite some time and surgical treatment of these lesions led to a relief of the headache. Because these two discussed points indicate that the revised 2004 IHS diagnostic criteria for headache attributed to rhinosinusitis do not catch patients with isolated sphenoid sinus lesion-associated headache, we suggest the necessity for an expansion and broadening of the IHS criteria to include patients such as those presented here who have headache only, and as in many other cases of sphenoid sinusitis without sinonasal symptomatology.

The diagnosis and treatment of sinonasal pathologies are relatively easy because symptoms and signs are obvious but in case of sphenoid sinus including isolated sphenoid fungal sinusitis due to lack of nasal symptoms initially these patients do not present to ENT surgeons but to neurologist, and neurosurgeon for vague symptoms, especially headache [6]. Physicians should be aware of this rare clinical entity and recommend complete rhinological workup in the intractable headache patient, as in many cases early diagnosis and appropriate treatment provide the key in achieving favorable outcomes [3].

Conflict of interest None.

\section{References}

1. Allphin AL, Strauss M, Abdul-Karim FW (1991) Allergic fungal sinusitis: problems in diagnosis and treatment. Laryngoscope 101:815-820 
2. Klossek JM, Serrano E, Péloquin L, Percodani J, Fontanel JP, Pessey JJ (1997) Functional endoscopic sinus surgery and 109 mycetomas of paranasal sinuses. Laryngoscope 107:112-117

3. Yiotakis I, Psarommatis I, Seggas I, Manolopoulos L, Ferekidis E, Adamopoulos G (1997) Isolated sphenoid sinus aspergillomas. Rhinology 35:136-139

4. Lee K, Yanagisawa K (1998) An obscure etiology for headache: sphenoid sinus disease. Yonsei Med J 29:209-218

5. Wyllie JW 3rd, Kern EB, Djalilian M (1973) Isolated sphenoid sinus lesions. Laryngoscope 83:1252-1265

6. Latif S, Aslam N, Ahmed R, Sarfraz S (2008) Isolated sphenoidal fungal sinusitis, presentation but potentially life threatening outcome. Annals 14:72-74
7. Lin SH, Ho CY (2006) Paranasal sinus pathologies in patients presenting with headache as the primary symptom. Cephalalgia 26(4):423-427

8. Bluementhal HJ (2001) Headaches and sinus disease. Headache 41:883-888

9. Headache Classification Subcommittee of the International Headache Society (2004) The international classification of Headache disorders: 2nd edn. Cephalalgia 24:9-160

10. Sellars SL, Devilliers JC (1981) The sphenoid sinus mucocele. J Laryngol Otol 95:493-502 